\title{
Sunburn-using excess energy of small-scale production for distributed computing
}

Nurminen, J. K.

2018-01

Nurminen , J K , Niemi , T , Strandman , J \& Ruokosuo , K 2018 , ' Sunburn-using excess energy of small-scale production for distributed computing ', Energy Efficiency , vol. 11 , no. 1 , pp. 97-119 . https://doi.org/10.1007/s12053-017-9552-1

http://hdl.handle.net/10138/321703

https://doi.org/10.1007/s12053-017-9552-1

acceptedVersion

Downloaded from Helda, University of Helsinki institutional repository.

This is an electronic reprint of the original article.

This reprint may differ from the original in pagination and typographic detail.

Please cite the original version. 


\title{
Sunburn - Using Excess Energy of Small Scale Production for Distributed Computing
}

\author{
J. K. Nurminen \\ Helsinki Institute of Physics, Finland \\ Aalto University, Finland E-mail: \\ jukka.k.nurminen@aalto.fi
}

T. Niemi

Helsinki Institute of Physics, CERN,Switzerland E-mail:

tapio.niemi@cern.ch

\section{J. Strandman}

Aalto University, Finland E-mail: jstrandman@gmail.com

\section{K. Ruokosuo \\ Aalto University, Finland E-mail:} konsta.ruokosuo@aalto.fi

the date of receipt and acceptance should be inserted later

\begin{abstract}
The Sunburn solar computer system is based on the idea of consuming the excess electricity of photovoltaic energy systems for useful computing. In this way, the proposed approach allows us to move a part of computing capacity from dedicated data centers to individual solar energy systems and thereby reduce the energy consumption of the data centers. In principle, the proposed mechanism converts the excess electrical energy into valuable computation. The results of computation or data processing are easier to store and transfer than produced electricity. In small scale systems, the benefit from storing or selling the excess electricity is small, whereas the benefit from selling the generated data is potentially larger, as we demonstrate in this study. Finally, the technical feasibility of the solution is illustrated by constructing and evaluating a prototype implementation using excess solar energy for distributed BOINC computing.
\end{abstract}

Keywords distributed computing · solar energy · green computing · energy storage $\cdot$ scientific computing $\cdot$ distributed data center

Address(es) of author(s) should be given 


\section{Introduction}

Home owners are increasingly installing solar panels and other renewable energy sources on their houses. While this may be a useful way to satisfy a part of domestic electricity need, the common problem of matching the supply and demand obviously arises. The case when the home needs more electricity than it produces is easy: simply buy the missing electricity from the grid. However, the opposite case when the home produces more electricity than it consumes, is more complicated. This is a new problem that has only arisen in the past few years. Typical solutions to the problem include selling extra electricity to the grid operator, storing extra electricity locally, or trying to time electricity consumption to better match production. In this paper, we study a new alternative: converting surplus solar production to computing results. The key idea is to equip the home with computing units and consume the excess electricity by performing computations.

The idea of adapting data center power consumption to the availability of renewable energy has already been investigated in, e.g., DC4Cities ${ }^{1}$ and Geyser $^{2}$ research projects. While the prior work has looked at use of renewable energy at data center level, we will take one step further and study the use of renewable energy for computing at household level.

Until recently, most of the heavy computing was done in computing clusters of companies or research institutes, while the lighter computing tasks were handled in the personal computers in offices and homes. With cloud computing technologies, computing is increasingly moving towards shared servers located in remote data centers. One of the enablers of this change has been the rapid development of Internet technologies, which allow fast and easy transfer of data to distant computing sites.

According to a recent report by Lawrence Berkeley National Laboratory [46] the data centers in United States consumed 70 billion $\mathrm{kWh}$ of electricity in 2014 , which is about $1.8 \%$ of total US electricity consumption. The consumption is increasing although with a lower rate than estimated earlier [29]. In any case the cost of electricity is among the most important components of computing. Therefore new ways to reduce the energy consumption of computing are important both for economic and for environmental point of view.

In this paper, we propose and analyze the idea of the Sunburn solar computer system. Note that although our focus in this paper is solar power, the same concept would work equally well with other power sources that have similar characteristics, such as wind power. Sunburn distributes computing to homes or other locations having solar panels for producing electricity primarily to own consumption. Because of large fluctuations in solar power production, these installations regularly produce more electricity than what is consumed locally. We call this over production excess energy and observe that its amount varies in time, sometimes in unpredictable ways. A fundamental observation

\footnotetext{
1 http://www.dc4cities.eu

2 http://www.geyser-project.eu
} 
is that excess energy has value and we have alternative ways to monetize it. In this paper our focus is to study the technical and economical opportunities to convert excess energy into results of computing tasks. The idea is based on a few observations and assumptions:

1. Computational results have monetary value, i.e. someone is willing to pay for computing.

2. It is possible to divide computational tasks into subtasks that can be distributed.

3. Moving data defining the computational tasks and the results is more efficient than moving electricity.

4. The price of computational devices continues to drop making it feasible to install computing capacity in homes.

5. Some computational tasks, e.g. in scientific computing, are not very time critical allowing delays that depend on availability of renewable electricity.

6. Some data and computations, e.g. in scientific computing, do not have very tight security requirements.

7. The computing needs continue increasing, for example because of the important role of computing in science, biotechnology, and other fields which rely on computational analysis.

Based on the idea of controlled energy consumption, complexities of the solar energy storage and energy transfer in general, the concept we present here is simple: Instead of selling or storing excess electricity produced by solar (or wind) energy systems, consume this electricity by using it for useful computations converting input data into results. The benefit of this is clear: by converting the electricity into data, we can avoid the difficult problem of energy storage and related inefficiencies. This approach can also be financially beneficial since processing data may be more profitable than selling the electricity to the grid. Since no electricity is sold to the grid, this also helps the grid company to avoid issues related to managing the fluctuating production of a huge number of small producers.

The aim of this paper is to describe the idea and share the experiences we have gained in implementing a prototype of the system. Briefly, the main contributions of this paper are:

1. We propose the idea of performing distributed computing at homes to consume the extra electricity generated at homes with solar or other renewable means.

2. We estimate the economical viability of the concept with a quantitative analysis based on solar power production in Finland, spot prices of Nordic electricity markets, and Amazon EC2 computing prices (Sections 3 and 4).

3. We study via our prototype implementation the technical issues in implementing the idea (Section 5).

4. We evaluate the feasibility of the idea with the measurements of our prototype, share some of the lessons learned and analyze the cost of the system (Section 6). 
5. We compare our idea with alternative ways of using the excess electricity (Section 2) and discuss the future potential of our idea (Section 7).

\section{Background and related work}

With the increasing implementation of renewable energy self-generation at homes, the mismatches in consumption and production create challenges for both electricity generators and grid operators. In the case of producing less than the demand, a simple solution is to buy the needed electricity from the grid. Because buying electricity from the grid has been the dominant paradigm, this requires no extra considerations. However, when a household produces more electricity than it consumes, the situation is more complicated [12]. In the following, we shortly analyze the different alternatives to manage the excess electricity.

\subsection{Curtailment}

A trivial way is to simply ignore the excessive electricity. This approach has a set of issues related to electric power distribution [55] and most importantly the unused power is wasted. It means that the capacity factor of the module is significantly reduced, weakening the investment made by the module owner. In households, the peak production (especially with solar) is during daytime hours when the house is empty, resulting in a negative correlation between consumption and production. In areas with high solar penetration, the majority of the module production would need to be curtailed [35]. Scheduling consumption tasks to be carried out when production is at its highest, can help to increase the correlation of demand profiles to production of solar panels. However, conventional demand-side management is limited to a small portion of a household's consumption [21] and therefore, although it helps to alleviate the issue, it cannot be considered as a solution on its own.

\subsection{Selling the electricity to a electric company}

Selling the overproduction back to the grid is a simple option from the household point of view. In this way, the household may get income from the extra electricity and the electricity companies can use the electricity wherever there is demand. The tariffs vary considerably depending on the region and current state of electricity markets as well as policy $[25,7]$. Since prices are currently subsidized in many countries, there is also an uncertainty what happens to the feeding tariffs in the future. In any case, buying electricity for anything higher than the spot market price is not sustainable from the electricity market's point of view. On the other hand, the compensation of excess electricity at the spot market price leads to negligible income for the module owner. 
From the transmission system operators' point of view, buying electricity from consumers is not trivial [48]. Even at relatively small levels of feed-in from households, voltage levels in the distribution grid can rise over capacity and cause problems in efficiency and put the grid at risk of a power outage. Grid-tie inverters are required to cut off the supply of electricity to the grid if voltage levels are too high, resulting in a forced curtailment which is undesirable. Ultimately, developing the grid to be capable of managing substantial amounts of grid feed-in would require large investments and increasingly complicated methods of system operation. To alleviate these problems smart grid technologies such as [50] have been developed.

\subsection{Selling the electricity to a local microgrid}

Selling the electricity to a microgrid [33] is similar to selling it back to electricity company, but the microgrid may yield a better margin for the sold electricity[30]. However, because the microgrid is often geographically small, the extra electricity production of different households can be highly correlated (e.g. clouds can block the whole neighborhood). Additionally, this solution obviously requires access to a microgrid, which is very seldom available.

\subsection{Store the excess electricity in batteries for later use}

Storing extra electricity in a battery is obviously an attractive alternative [58, 38]. For instance, the Tesla Powerwall solution aims at providing an electricity storage to households. This approach is easy to implement in a small scale, good for off-grid situations, and it also solves the overproduction problem if sufficient storage capacity exists. Implementing storage capacities large enough to account for the excess electricity of solar panels would not only be extremely expensive but would also cause losses due to both self-discharge and the conversion of electricity to chemical energy and vice versa [13]. The production of electrical storages is also energy intensive and environmentally questionable, which directly contradicts the pursuit of environmentally friendly energy through solar panels [31].

\subsection{Using the electricity to process data and sell the data}

Finally, the approach that we are proposing in this paper, is to consume the extra electricity immediately as it is generated by performing computations. The idea is based on an assumption that data is potentially easier to transfer than electricity and a computation task can be activated at any time from a backlog of computing jobs. In a financial sense, we must assume that the value of processed data is higher than the value of an alternative usage of the electricity. The benefits need to be balanced against the potential problems: the cost and useful lifetime of computer hardware and the degree of computer 
utilization during the lifetime. Furthermore, because the computing platform is only intermittently available in this kind of scenario, the suitable workload is limited. Interactive real time applications are clearly not suitable but we see a large potential, for example, in scientific computing and computationally intensive simulation and optimization task in industry. Moreover, workloads from voluntary computing platforms, such as SETI@home [2], would be suitable for this purpose.

The idea for intentional and controlled energy consumption was originally investigated in our earlier work [39] regarding using computers as heating units. Lie et al. [34], as well as some startup companies (e.g. Cloud \& Heat, Qarnot, and Nerdalize), have worked with the idea of computing heaters where the computer and its CPU usage is controlled in order to maintain a desired room temperature. Although the technical solution of our present paper is able to apply some of the ideas of these studies, the target in the current study is completely different. Instead of starting the computing when the house or water needs heating, we control the computing by the amount of excess electricity available in the household.

A number of studies have investigated the opportunities to move computing to follow the availability of energy. Van Heddeghem et al. [23] study how distributed computing can be used to reduce carbon footprint by using renewable energy. To deal with the problem of unpredictable availability, the authors propose moving the workload to data centers having renewable energy available at the moment. A similar idea is used by Le and Wright, who aim at reducing electricity bill [32] by optimally scheduling workload to different data centers based on the price of carbon emissions and electricity. The idea of 'mini data centers' of Bird et al. [6] is based on a concept of using multiple distributed small data centers for improving usage of renewable energy. Rahman et al. [42] study geographic load balancing methods for data centers in the smart grid. On the other hand, Zheng and Cai [59] study how online service providers can minimise their electricity consumption by using an energyaware load dispatching method among data centers in different geographical locations. Anghel et al. [3] have a slightly similar approach by processing computational tasks in a data center also producing renewable energy. Cioara et al. [9] have studied by simulations how a data center connected to a smart grid could improve its profitability and energy efficiency by shifting workload in time or using its backup power for energy production based on the energy supply and demand situation at the moment. In comparison to our work, these approaches are primarily looking at how to perform the computing in energyoptimal locations while we study what can be done with the excess electricity generated by households and propose to use it for computing. Naturally, these two perspectives complement each other. Moreover, most of the prior work is conceptual. Instead, in our work we bring the idea closer to practice by building a prototype system.

Another set of studies has investigated green data centres. For instance, Kong and Liu [28] review green and energy-aware power management methods for data centers. They identify several methods: work load scheduling, virtual 
machine management, and energy capacity planning. Dupont et al. [14] propose a virtual machine placement algorithm, which can consider multiple constraints including energy within a constraint programming based approach. Ye et al. [57] introduce an energy storage system for green data centers. By storing electricity, a data center can better handle peaks in power demand and also utilise renewable solar or wind energy. Other projects, such as the Parasol research project, have tried to maximize the use of green electricity in data centers [20]. Finally, Yang and Chien [56] have proposed to use excess renewable energy to run data centers. Pernici et al. [41] have developed GAMES approach containing green performance indicators for optimising energy efficiency.

Finally, there are several studies on computational platforms for real-time operation such as [19], middleware solutions for improving communication [17], tools to construct flexible and distributed computing platforms [53,54]. Applying these approaches to our system would increase possible workload types it can process. In their recent work, García-Valls and Calva-Urrego [18] introduce a powerful middleware layer being aware of multicore architectures. This can significantly improve the performance since in practice all current CPUs have several cores.

\section{Economic analysis of alternative ways to consume excess electricity}

In this section, we present tentative calculations to estimate the economic viability of our concept. Rather than giving a detailed analysis, our purpose is to roughly understand the feasibility of the new concept and the factors influencing its feasibility. Therefore, we do not, e.g., consider necessary investments, amortizations, and other factors in detail but this quantitative analysis mainly aims at supporting the qualitative discussion of the possibilities. To make the discussion concrete we use Finland as an example case. Applying different values which reflect the different conditions in other countries should be straightforward for the reader.

\subsection{General model}

A simple model allows us to compare the value of the computing results $P_{\text {comp }}(E)$ with alternative uses of the same excess energy. In the analysis we consider three alternative scenarios to generate profit: curtailment $\left(P_{\text {cur }}\right)$, selling the electricity to the grid or microgrid $\left(P_{f e e d}\right)$, and storing the electricity in a local energy storage $\left(P_{\text {store }}\right)$. Each alternative requires some investment to equipment and some maintenance. Both of these costs are included in the investment costs $C_{\text {comp }}, C_{\text {feed }}, C_{\text {store. }}$. Then the benefit, $U$, of using the excess energy for computing and selling the computing results in comparison to alternative uses of the energy is: 


$$
U=\left(P_{\text {comp }}(E)-C_{\text {comp }}\right)-\left(P_{\text {other }}(E)-C_{\text {other }}\right),
$$

where $P_{\text {other }}$ and $C_{\text {other }}$ are the profits and costs of alternative uses for the excess electricity (curtailment, feed-in, and storage). The computing cost also includes the depreciation cost of the computing hardware. Table 1 summarizes the essential differences between the alternatives.

Table 1 Summary of alternative costs

\begin{tabular}{|c|c|c|}
\hline Approach & Income $(P)$ & Cost $(C)$ \\
\hline Computing & value of computing results & computing system \\
Curtailment & no income & none or minor \\
Feed-in & value of feed-in electricity & feed-in equipment \\
Storage & savings in electricity purchase & storage system \\
\hline
\end{tabular}

\subsection{Curtailment}

We assume that curtailment does not generate any income, $P_{\text {cur }}=0$. In fact some additional equipment may also be needed for the curtailment case. Curtailment may also require some parameters of the photovoltaic system to be set to non-optimal values thus a small cost can also be present in this case. Therefore $C_{\text {cur }}>0$, but the cost will be minor.

\subsection{Feed-in}

In feed-in case, $P_{\text {feed }}$ depends on the electricity pricing. Currently, in countries such as Finland, compensation of small scale production is based on the spot prices on the Nordic electricity markets. These prices fluctuate based on demand and supply of the electricity, with the typical price being between 2.5 and 4 euro cents per kWh for most Nordic countries as illustrated in Fig. 1.

In comparison, the consumer price of electricity includes tax and usually some form of transfer cost, which brings the overall price higher. In the case of Finland, the price has been around 12 and 13 cents per kWh.

Because the price of sold electricity is so much smaller than its purchase price, selling produced excess electricity is not a very profitable venture. If any additional costs, such as sale tax, are added to the price, the profits are reduced even further.

\subsection{Storage}

By storing the electricity it is possible to reduce the amount of electricity which needs to be purchased. For instance, the day time production of solar 
2014 Elspot prices in EUR/MWh

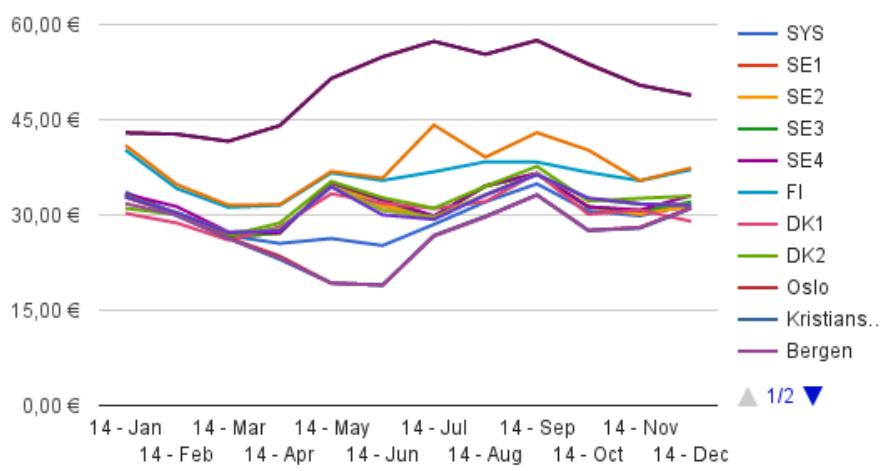

Fig. 1 An example of variations in spot prices in Nordic electricity markets. These prices reflect the value of electricity sold back to the grid. Lines represent different locations. (http://www.nordpoolspot.com/)

electricity can be used at night time. This obviously reduces the energy bill. On the other hand, the price $(\$ 3000$ for $5.6 \mathrm{kWh})$ is so high that it remains open if the investment would be profitable $[45,51]$.

\subsection{Computing}

Like energy storage above, the computing case has a lot of uncertainties and is subject to fluctuations in prices. This is further complicated by the fact that there are no prior examples of services which provide large scale computing services in intermittent way. Obviously computing service which is only occasionally available is suitable only for some scenarios. The unpredictable availability of the service will naturally influence its price. On the other hand, in the most of the cases, it is possible to increase availability by using grid electricity for running computing resources when solar energy is not available.

To estimate the value of computing, we use Amazon EC2 as a comparison point. From EC2 it is possible to purchase computing power by an hourly rate. The service has multiple hardware options which have a big influence on price and computing power. Table 2 shows a snapshot of Amazon AWS on-demand instance prices. The Amazon pricing model $^{3}$ has a lot of alternatives and varies in time, so the table reflecting prices at January 2016 only illustrates the pricing levels of computing. ECU is Amazon's own relative value reflecting the computational capability of the instance. The hourly price of the computing when purchased from Amazon would thus be ranging from $0.0137 €$ to 0.137 $€(\$ 0.015$ to $\$ 0.15)$.

3 https://aws.amazon.com/ec2/pricing/ 
Table 2 Examples of Amazon EC2 computing prices (Jan, 2016)

\begin{tabular}{|c|c|c|}
\hline Type & ECU & Price \\
\hline t2.micro & variable & $\$ 0.015$ \\
t2.medium & variable & $\$ 0.03$ \\
t2.large & variable & $\$ 0.06$ \\
m4.large & 6.5 & $\$ 0.143$ \\
m4.xlarge & 13 & $\$ 0.285$ \\
\hline
\end{tabular}

To estimate the value to intermittently available computing, we can look at the spot market of computing. For instance Amazon is auctioning its excess resources and studies estimate dramatic saving opportunities via them [49]. The Amazon spot pricing is complicated but analysis of different Amazon markets [49] shows that the price of a spot instance can be as low as $5-10 \%$ of the regular price of the same instance. However, there is big volatility and variability in the availability of such instances. The service sensitivity to delays can potentially be characterised with extended service level agreements as proposed in [15]. Their service flexibility agreement concept would allow specifying the tolerances for delays and in that way connecting different computing costs to different levels of flexibility. Quantifying the dependency is a topic for further research.

Purchasing computing power from individual household has further limitations including security and privacy issues. Moreover data transfer can be a bottleneck if big amounts of data are needed for the computing. At present, the active distributed computing activities such as BOINC, seti@home or Mersenne prime search are all based on voluntary work and there is no monetary incentive. Furthermore, Mukherjee et al. [37] have developed a framework which allows ad-hoc sharing of unused computing resources with monetary compensation. However, their dynamic pricing and incentivization model is rather abstract providing little help in what could be realistic prices.

Matching the computing power of our test implementation with EC2 instances is not straightforward, since EC2 instances are virtual machines running on top of different hardware and there is no guarantee what specific processor the instance is running on [40]. However, we can safely estimate that the computing power of a single computing unit we have built from regular off-the-shelf components is ranging from t2.micro to m4.large. Our prototype uses Intel Core I3 processor while Amazon EC2 is using Intel Xeon family processors $^{4}$. The performance of these is rather close to each other (i3 is slightly better in single-core benchmark while Xeon is slightly better in all-core benchmark) [10].

To provide this computing power we need the original investment for the necessary equipment (which we analyze in Section 6.4) and the electricity it consumes. Assuming that our computing unit consumes $50 \mathrm{~W}$ (this is the upper limit for our prototype), we can power 20 such units with $1 \mathrm{~kW}$. This

4 https://aws.amazon.com/ec2/instance-types/ 
means that the value of selling the computing results of $1 \mathrm{kWh}$ consumed in computing, is ranging from $0.27 €$ to $2.7 €$. As discussed above, the value of electricity sold back to the grid would only be in the range of $0.025 €$ to 0.04 $€$. Thus, selling computing results instead of electricity would be much more profitable. Note that the computing offered in this way is not as predictable and reliable as what EC2 or other cloud services offer but because the high price gap, we have some room to compensate for this in pricing.

\section{Annual analysis with seasonal variations}

The above calculation shows that selling computing is clearly superior to selling electricity. However, it is not taking into account the fact that there are big seasonal variations in the available solar energy. In this section, we present a more holistic analysis comparing computing with other uses of excess electricity over the timespan of a whole year. Because the analysis requires assumptions of regionally varying and uncertain parameters, we make the model available for the interested readers to play with. ${ }^{5}$

We will investigate the following four scenarios:

1. Computing with excess energy (subsection 4.1)

2. Computing with excess energy complemented with grid energy (subsection 4.1)

3. Computing for heating (subsection 4.2)

4. Combined computing and heating (subsection 4.3)

Using the same example calculations as above, insolation in northern countries would allow substantial amounts of PV overproduction from April through August and decent amounts during March and September, yet very little outside of that (See Fig. 2). The solar irradiance values are average monthly values 6 .

The computing module is most applicable in scenarios where the arrayto-load ratio (ALR) is high. In our calculations, an ALR value of 8 is used, meaning the panels would generate roughly the same amount of electricity in one year as was consumed during the same year. If we wanted to consume nearly all of the excess production, we would need a computing module capable of consuming 4500 watts of power. This would not be economic, as the PV system produces its nominal output for only a marginal part of the year, which would cause the computing module to have a very low utilization rate. Therefore, we choose the maximum power consumption of the CPU module to be to be $1 \mathrm{~kW}$, allowing it to consume the majority of excess production. As the heat generated by the CPU module is an inconvenience during summer

\footnotetext{
5 The detailed parameters and calculations can be found in https://docs.google.com/ spreadsheets/d/1YQxgcIxVAgqZZITZ-fvIMWjE-3n3K3vSDEAuXjLyiU4/edit?usp=sharing. The interested reader can also explore the effect of different assumptions with the model by changing its parameters.

6 http://www.efficientenergysaving.co.uk/solar-irradiance-calculator.html
} 


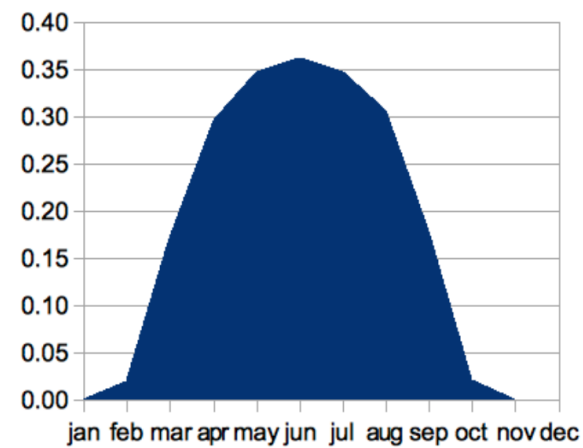

Fig. 2 Monthly utilization rates of when using excess electricity

Table 3 Values for solar power estimation

\begin{tabular}{|l|l|}
\hline Annual consumption & $4600 \mathrm{kWh}$ \\
Retail price of electricity & $0.1 € / \mathrm{kWh}$ \\
Compensation for feed-in & $0.04 € / \mathrm{kWh}$ \\
Computing unit price & $6000 €$ \\
Computing unit capacity & $20 \times 50 \mathrm{~W}$ \\
Computing unit lifetime & 5 \\
Computing unit lifetime for heating & 4 \\
Solar module nominal power & $5 \mathrm{~kW}$ \\
Annual generation & $4350 \mathrm{kWh}$ \\
Solar module price & $9000 €$ \\
Solar fraction & 0.35 \\
Battery capacity & $7 \mathrm{kWh}$ \\
Battery Price & $3000 €$ \\
Cooling component price & $1500 €$ \\
Cooling component capacity & $4500 \mathrm{kWh}$ \\
\hline
\end{tabular}

months, we also included a cooling unit in the system. The calculation assumes the lifetime of the system to be five years. Other relevant variables used in calculations are gathered in Table 3 .

\subsection{Computing with excess energy with and without grid support}

The economic viability of the proposed system can be estimated by calculating a profitability limit for computing. If the compensation for computing exceeds the limit then it is advantageous to use the excess electricity for computing in comparison to the alternative scenario. In Table 4, we compare computing with four different alternative cases:

1. no PV production and no computing at all,

2. excess electricity is stored in local energy storage,

3. excess electricity is sold with feed-in tariff, and

4. the panel size is reduced to cut the amount of spare electricity generated. 
In addition to these cases, the columns in Table 4 show two computing cases. First, only excess PV production is used for computing resulting into only $17 \%$ utilization rate for the computing units. Second, excess PV production is complemented with grid electricity to reach $50 \%$ utilization for the computing units. In this case $34 \%$ of electricity is supplied with PV and the rest is purchased from the grid. When the computing unit uses only PV electricity, its variable costs are nearly zero. However, the initial investment cost is still rather high. To compensate this a high utilization rate would be useful. Therefore, the alternative to complement the PV production with grid electricity seems attractive. For instance, the comparison with the case without PV production shows that if computing brings value that is higher than $0.36 € / \mathrm{kWh}$ then the system is economically profitable if also grid electricity is used for computing. Using only the excess PV energy, the limit is $0.70 € / \mathrm{kWh}$. Since the value of computing results can be in the range of $0.27 € / \mathrm{kWh}$ to $2.7 € / \mathrm{kWh}$ (as we discussed in previous subsection), the concept can be economically feasible.

Table 4 Profitability limits $€ / \mathrm{kWh}$ for computing with excess electricity in comparison to alternatives

\begin{tabular}{|c|c|c|}
\hline Alternative method & Excess PV only & Excess PV and grid \\
\hline no PV, no computing & 0.70 & 0.36 \\
electrical storage & 0.48 & 0.26 \\
grid feed-in & 0.63 & 0.33 \\
downsizing panels & 0.69 & 0.36 \\
\hline
\end{tabular}

\subsection{Computing for heating}

Maintaining sufficient utilization rates is vital for providing stable enough computing service and for increasing the profitability of the investment. In the previous calculation, the utilization rate of the computing module with excess electricity only, would be good during summer months, exceeding $30 \%$ between April and August. However, in Nordic conditions, utilization would be nearly zero for a large portion of the year, giving a yearly utilization rate of around $17 \%$. To avoid long periods of shutdown, we assumed to use also grid electricity for computing during winter periods. The computer produces heat at a conversion rate similar to the resistive electric heater, providing an easily controllable source of heating. In this way heating is a by-product of computing or, depending on the point of view, computing is a by-product of heating.

In this subsection, we first analyze the case where the computing is used for heating only. This case has already been investigated earlier [34] and early commercialization activities are already ongoing. In the next subsection we investigate the case of joint heating and computing. 
Utilization of computing for heating allows for sizable utilization rates, as the demand for heat during winter periods is very high. In this case heat is almost continuously needed and the amount of computing capacity is the bottleneck. Assuming a technical maximum utilization rate of $75 \%$, the annual utilization rate is around $48 \%$. Note that this high utilization rates are only possible in cool climate zones, like in the Nordic countries and parts of North America. Higher utilization rates could naturally make computing even more profitable.

The first row of Table 5 shows the profitability limits when computers are only used to generate heat. The electricity consumed by the computer is entirely converted into heat, producing the computational value as a "sideeffect". Alternative ways of heating vary in their efficiency. The efficiency is characterized by the coefficient of performance (COP), which indicates how many units of heat are provided by one unit of electricity. In this analysis we compare the computing for heating with cases where $C O P=1,2,3$.

The values on the first row of Table 5 show that using the computing units for heating is not as attractive as utilizing excess PV electricity for computing. In comparison to the profitability limit of $0.36 € / \mathrm{kWh}$ of "no $\mathrm{PV}$, no computing" case in Table 4 when computing was used to consume excess electricity with some grid support, using computing for heating is less profitable. Additionally, using computing for heating could be challenging to deploy due to additional systems installations required to distribute the heat properly throughout the house.

\subsection{Combined computing with excess energy and computing for heating}

In the final case, we use the same example values as above but now the computing module is used for both the consumption of excess electricity and for heat generation. It provides highly economic operation, maintaining utilization rates above $30 \%$ for each month of the year. The overall yearly utilization rate is $59 \%$ (in comparison to $17 \%$ when used only for consuming the excess electricity and $48 \%$ when used for heating only). Monthly averages are shown in Fig 3.

Table 5 Profitability limits $€ / \mathrm{kWh}$ for heating and combined cases (computing both for spending excess electricity and for heating) in comparison to alternatives

\begin{tabular}{|c|c|c|c|}
\hline Alternative method & COP 1 & COP 2 & COP 3 \\
\hline Only heating & 0.60 & 0.65 & 0.67 \\
No PV, no computing & 0.22 & 0.26 & 0.27 \\
electrical storage & 0.16 & 0.20 & 0.21 \\
grid feed-in & 0.20 & 0.24 & 0.25 \\
downsizing panels & 0.22 & 0.26 & 0.27 \\
\hline
\end{tabular}

Table 5 shows similar comparison to alternative uses of excess electricity as Table 4. By comparing those two tables, we can clearly see that the combined 
case is profitable with far smaller compensations from the computing. Also, all values are below or equal to the monetary value of the computing that we estimated to be in the range of $0.27 € / \mathrm{kWh}$ to $2.7 € / \mathrm{kWh}$ (as we discuss in Subsection 4). The different COP values have some but not a very significant effect.

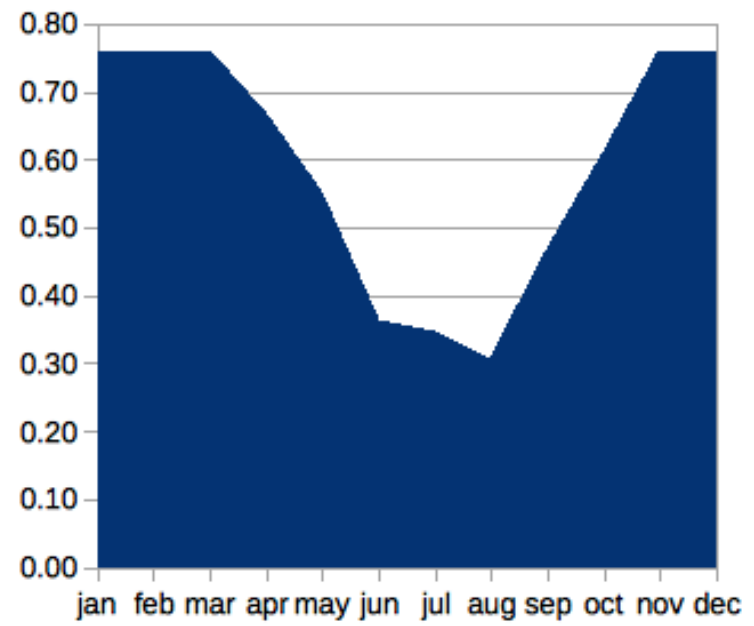

Fig. 3 Monthly utilization rates of when using excess electricity and heating

\section{Prototype implementation}

To better understand what it would take to build and use the system, we developed a small-scale prototype using standard off-the-shelf components. It consists of a normal solar energy system, a computing unit, and a control unit. Naturally, this prototype cannot be used to fully evaluate the whole concept but it is sufficient to show that required hardware is possible to build with a reasonable cost. The control system has sensors for current and voltage measurement. These are used to measure the energy balance of the overall system and identify the amount of excess energy the system has available at any point of time. The control system operates and controls a separate computing unit, which consumes the excess electricity by processing data. The amount of electricity consumed by the computing unit is controlled by limiting the number of active CPU cores and their workloads. The control signals and all the processed data are transferred using a standard Ethernet network and Internet connection.

The workload for the prototype is provided by distributed computing projects of the BOINC platform [1]. As the tasks provided are real world problems, we get a reasonably accurate picture how well computer performs on such tasks without need for approximation based on artificial loads. 


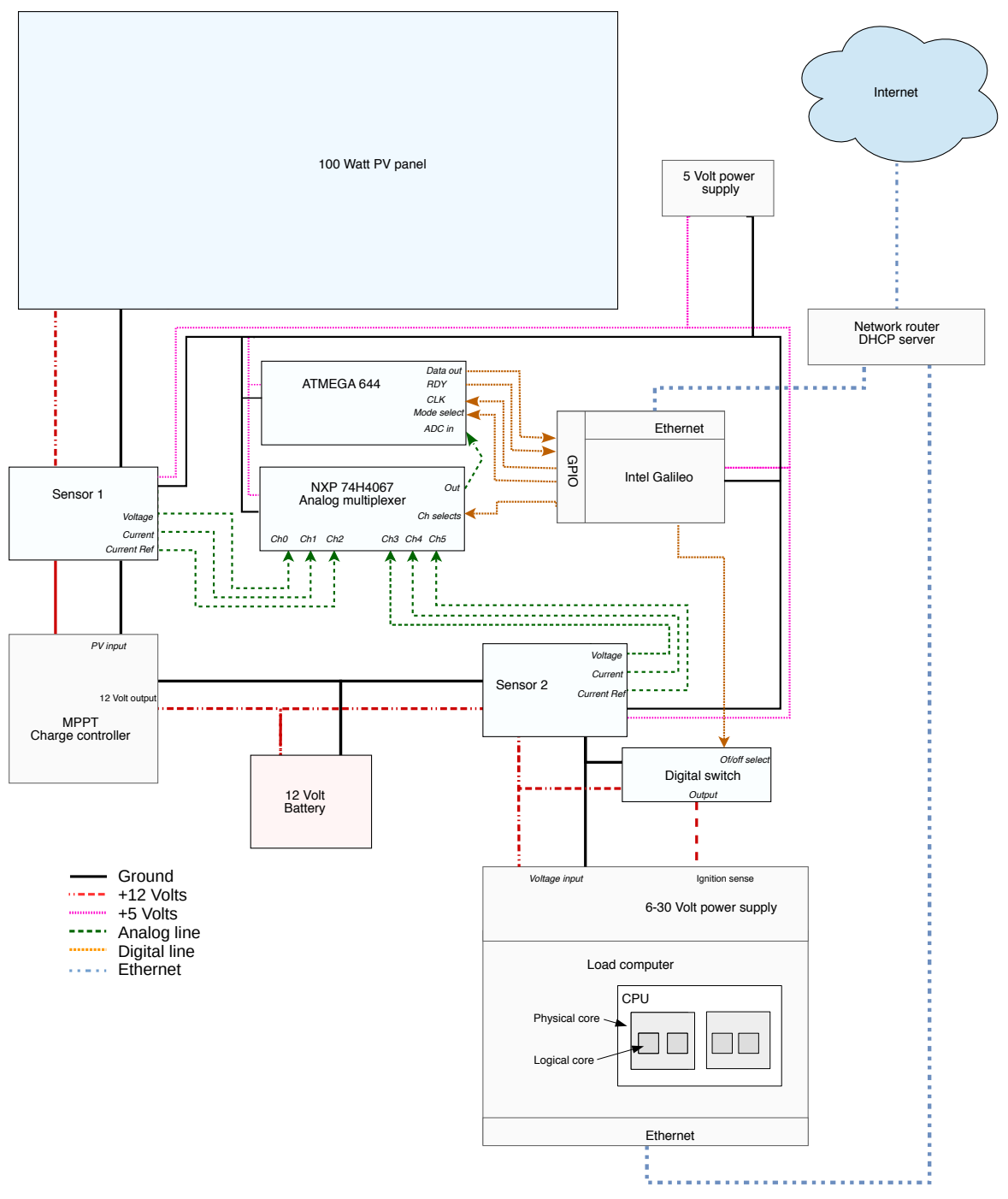

Fig. 4 Schematic diagram of the Sunburn prototype

\subsection{Technical overview}

As shown in Fig. 4, the main components of the system are the control unit, sensors for measuring both voltage and current, multicore computing unit (Load computer) and the solar energy system. The controller is based on the first generation Intel Galileo development board, which handles the data from the sensors and performs all the control operations in the system. These include the shutdown and startup of the computing unit, calculation of the CPU/core limits and data logging. The Galileo board was selected based on the low power 
consumption and analog and digital connection pins, which allow interfacing with lower level electronics and sensors of the system. The other components of the control unit consist of additional boards for interfacing with sensors and switching circuit to control the power switch of the power supply of the computing unit.

The system has voltage and current sensors to determine the power output of the solar energy system (Sensor 1 in Fig. 4) and the power consumption of the computer (Sensor 2 in Fig. 4). The sensors are a combination of hall-effect transducers for current and voltage dividers for voltage. The sensor outputs a voltage reading in range of $0-5$ volts, which is read using analog to digital converter. In early phases of the system development, A-D conversion was done by the ADC chip on the Galileo board and in later phases by a higher quality ADC on a separate Atmel ATmega 644 powered interface board. This additional interface board was combined with an analog multiplexer to provide for future scalability and to ensure the quality of the measurements. While the actual measurements were performed by the ATMega microcontroller, the control of the multiplexer was left to Intel Galileo, partially due to pin limitations. The complete connection diagram of the sensors and control system is presented in Fig. 4.

The computing unit (Load computer in Fig. 4) in the prototype is a generic x86 computer built from off-the-shelf components, selected with reasonable performance and power consumption in mind. It has an Intel Core I3 quad core processor with a thermal design power (TDP) of about 50 watts. The overall design in independent of the processor selection; any reasonable x86 processor could be used. The processor is combined with SSD hard drive and a power supply designed to operate with direct current in the range of 6-30 volts. The power supply makes it possible to operate the computer directly from the DC power supplied by the solar energy system and attached batteries and thus reduces the overheads caused by voltage conversions to normal alternating current. Additional benefit from the DC-DC power supply is its high efficiency of 95 percent at typical loads. This is mostly due to close proximity of the battery operating voltages and computer voltages.

The operating system used is a Debian Linux distribution intended for server use. Because we designed the system to run on generic x86 computer hardware, both hardware and software of the computing unit can be flexibly altered to suit different situations and needs.

The final component of the prototype system is the solar energy unit. We developed it to match the computing power of the computing unit. (In real use both more solar power from bigger panels would be available and multiple computing units would be used to consume the electricity.) The solar energy system consists of a 85 watt polycrystalline panel connected to Maximum Power Point Tracking (MPPT) charge controller. A small battery provides power for the charge controller and it is also used to power the computing units for a few seconds to shut it down in controlled fashion in the case electricity supply drops suddenly. The panel was chosen to provide maximum energy output of almost double the consumption of the computer to ensure sufficient 


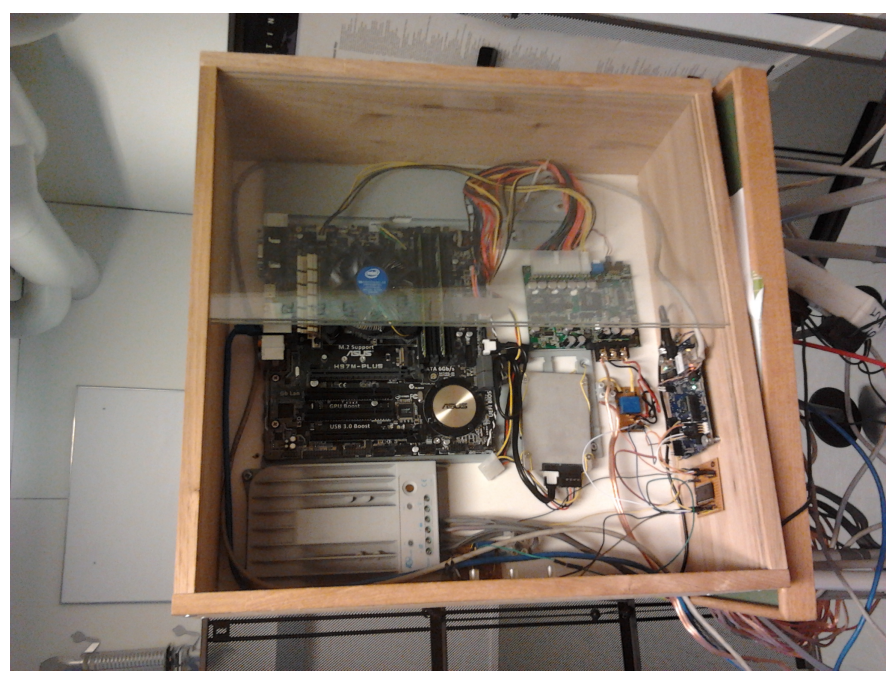

Fig. 5 Picture of the prototype system

energy for the testing during the low irradiance months of Finnish autumn and winter. For the same reason, the MPPT controller was selected for the system over the normal PWM charge controllers as the former try to maintain the ideal voltage and current values of the connected PV panels and thus optimize the energy output. The solar energy unit was connected to an ordinary car battery during the testing but a far smaller battery would have been enough. In principle, any normal 12 volt battery should work with the system.

The complete prototype system after assembly is shown in Fig. 5. Note that the prototype system has not been optimized. For instance, it could be made far smaller in a real implementation.

\subsection{Control System}

As mentioned earlier, the control system uses a combination of an analog multiplexer and ATMega microcontroller to measure the signals from the different sensors. Each signal is first selected via the analog multiplexer and then this signal is converted to the digital format using the ADC on the ATMega microcontroller. As the switching of multiplexer and the communication with the ATMega add some overhead, it makes sense to perform several measurements and average the result on the ATmega before transferring the collected data to the Galileo. Performing several ADC conversions also reduces measurement errors from minute fluctuations in the measured signal. As some of the measured signals are more stable than others, two different measurement variants were implemented on the ATMega. A short measurement, in which the measurements are performed consecutively for about 150 milliseconds, is used for stable signals to reduce the overall measurement time. A longer measurement 
of about one second is used on the potentially fluctuating signals, especially the current measurement signals. This longer time window of one second is based mainly on the behavior of the computing unit and more specifically of the used CPUlimit program.

The CPUlimit program controls the CPU usage and thus the electricity consumption. It works by suspending and resuming processes in order to achieve the given target CPU utilization level. The control period of CPUlimit is one second and the execution time of controlled process is a fraction of this period, determined by the desired limit percentage. Thus at $50 \%$ limit, a controlled process would execute for 500 milliseconds during each one second control period. The result of this is that during each of the control periods, the CPU core electricity usage fluctuates between maximum and idle power consumption, depending whether a process is running on the core or not. As a single measurement does not give us complete picture on the current power usage of the CPU, a longer measurement time is needed. The obvious choice is to use time close to one second, which matches the control period of the CPUlimit and should give a good average on the processor power usage.

\subsection{Operation}

Fig. 6 shows the typical operation of the system. It begins an operation cycle by performing the necessary measurements for decision making. After the measurements are performed, the system calculates the solar and computer system current power values and assesses if sufficient amount of power is available to operate the computer. If sufficient solar power is available, then a CPU adjustment is performed using a PID controller and measured core power limits. As each core in the processor consumes a given amount of electricity at maximum utilization, the power consumption for each core can be measured and used to choose a suitable amount of cores to be active. This sets the computer system close to the intended power target easily. To fine-tune the accuracy, a PID controller is used to adjust the CPU utilization close to the correct level and to adjust for fluctuations in the power values. To do so, the power output of the solar system is used as the PID target and the current power consumption of the computer system is given as the present state for the PID. The result given by the PID controller is then added to the current CPU limit. Both the $\mathrm{CPU}$ and core limits are then sent to the computing unit, where the client software limits the processes accordingly.

If there is insufficient amount of electricity available, the computer system is powered down both via control signals to the client software and by using the digital power switch connected to the power supply. As the power supply is signaled to shut down, it sends an electrical shutdown signal to the connected computer and starts a timer that will eventually shut down the system forcibly in case the computer fails to respond. This hardware and software combination shortens the shutdown time by few seconds, but most importantly ensures 


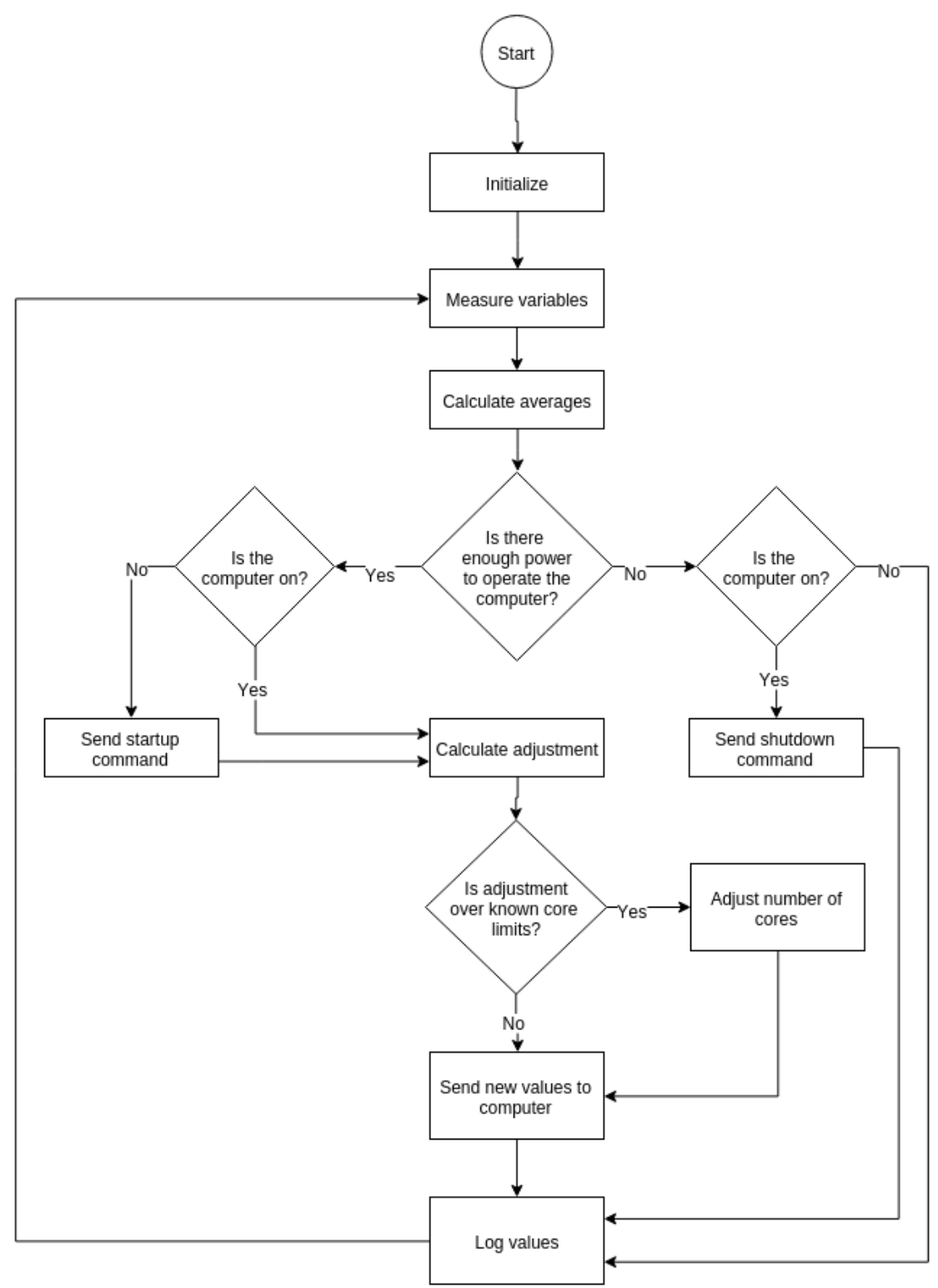

Fig. 6 Flowchart of the operation of the system

that there is maximum amount of time for the system to perform gracious shutdown.

After performing all the main operations, the control loop stores the measured values into log files that are used to monitor the system operation and performance. The system then begins a new loop, which performs most of the same operations. Because there are some time lags in computer responses, the 


\section{Core utilization progression - Model 1}

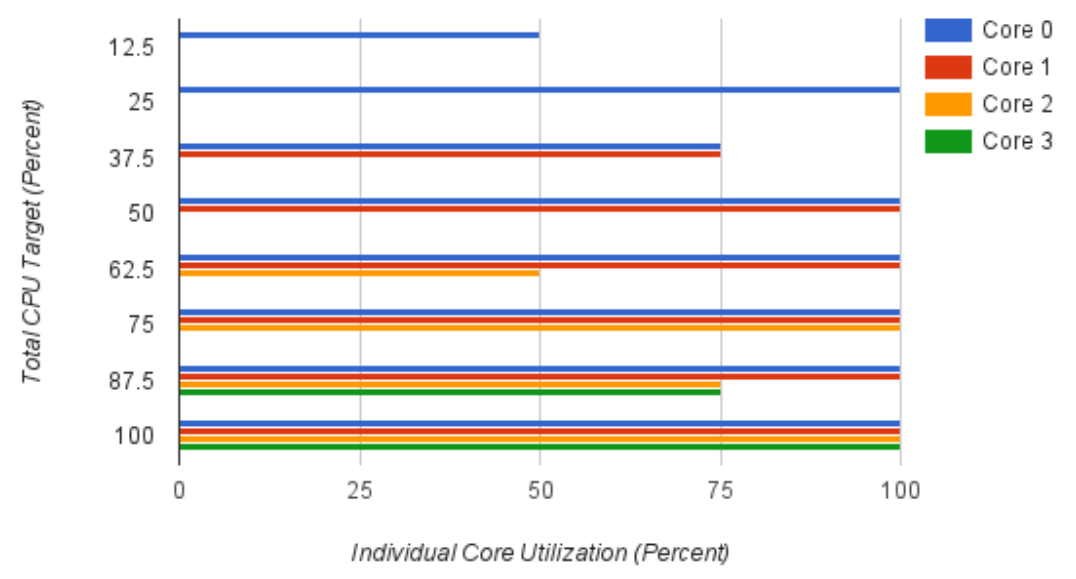

Fig. 7 Core utilization as a function of CPU load - Basic strategy. The lines are in the same order as given in the legend.

adjustment phase is only performed once in every three iterations to prevent the system from needlessly causing oscillations by excessive adjustments.

On the computing unit, the received control values are passed on the CPUlimit program that performs the actual limiting of the target processes. In addition, as the prototype system uses BOINC distributed computing platform [1] to provide the computational workload, the BOINC is also instructed to use only certain number of cores and thus the number of controlled processes is automatically reduced to a suitable number. This gives a small performance gain, as each CPUlimit instance is only capable of limiting the target process to minimum of 7 percent in real use scenarios.

The approach of selecting cores to be used is illustrated in Fig. 7. The essence of this approach it to keep the minimum number of cores active. Because the energy saving measures used in Intel Core processors allow the cores to enter power saving modes individually, the physical cores are activated in order to maintain as many cores idle as possible. The Core i3 processors support Intel HyperThreading technology which allows two threads to be executed simultaneously on each core. From operating system viewpoint, each physical core on the CPU has two virtual cores that behave in similar fashion as normal cores. To efficiently use this technology in active core selection, both virtual cores of a physical core are activated first before moving to virtual cores of other physical cores.

An alternative strategy to core utilization would be to use both of the virtual cores at the same time as illustrated in Fig. 8. This alternate model could lead to better performance as HyperThreading tries to maximize the use 


\section{Core Utilization Progression - Model 2}

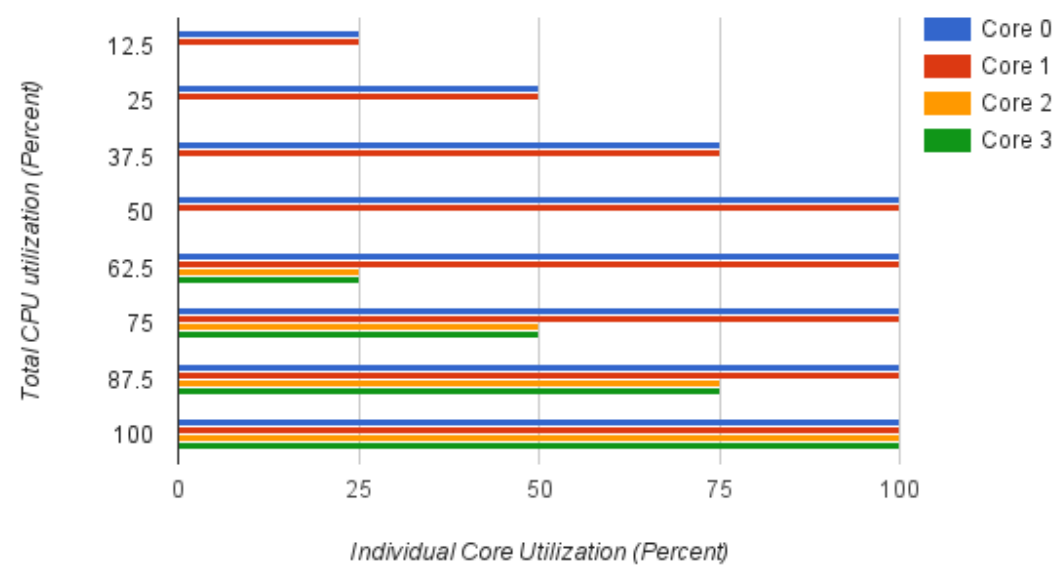

Fig. 8 Core utilization as a function of CPU load - Alternative strategy. The lines are in the same order as given in the legend.

of core cycles. The caveat is that in this approach the controlling of the processes is less effective and more prone to fluctuations, leading to less accurate energy usage. Thus, the first control model is used in the prototype system as a compromise between efficient core usage and control accuracy.

\section{Lessons learned}

\subsection{Control and sensory systems}

Testing the components of the system was done in three separate phases. Phases one and two focused on the sensor operations and the load control respectively, whereas the third phase tested the system as whole and connected to the solar energy system.

The voltage and current measurement tests were performed until the measurements consistently provided accurate enough results to be relied upon when the system would be connected to actual solar power systems with limited power. Main issues solved during the testing were the measurement of the power consumption of the computer when the CPU utilization was limited. When restricted to less than full utilization, the scheduling algorithm limit the power usage by pausing and resuming the execution of the computing workload until a suitable utilization value is reached. The result of this is that a single measurement would indicate either a maximum power or idle power consumption for the computer, depending on whether the process was waiting or executing at the moment. The solution was naturally to perform 
a several consecutive sensor reads and calculate the average of these reads to gain more accurate value. With fine tuning method, the system was able to produce readings matching ones measured with separate voltage and power meters.

The control testing involved calibrating the mechanism limiting and controlling the CPU. The main challenges were in tuning the control to encompass the multiple cores and hyper-threading capabilities found in multicore processors. This included optimizations to ensure that each controlled process would execute on certain cores, such that the physical and virtual core tied to it would be fully utilized before utilizing the next physical core. The control testing also involved calibration and preliminary testing of the PID controller. At the end of these tests with the early prototype, the control of the system worked well enough, taken into account the accuracy of sensors.

The scheduling of the workload described above and in Section 5.3 takes care of adapting the computing load to the available power. In an operational system this detailed level scheduling would need to be complemented with higher level scheduling. The higher level scheduling would manage the computing at job level and decide when and where each job will be computed. The jobs could e.g. have priorities and deadlines, and the system would then assign them appropriately for computing [8]. Also existing studies on scheduling in volunteer computing could be adapted for optimizing the scheduling [5, 52 ]. While this is regular practice in job queue management systems, in our case the problem becomes more complicated. First, we should use historical data of computing availability as well as weather forecasts to estimate how much computing resources will be available in each home and then use this knowledge as a basis for scheduling. Moreover, the system would need to have a mechanism to move a job to a new home if it does not have available resources now or in forecasted future [47,27]. Naturally, unnecessary moving of jobs is also wasteful for resource use. Therefore we would need to balance the amount of job movement with the availability of resources. A fair scheduling mechanism in this complex environment is a challenge for further work.

\subsection{Integrated system}

In the integration testing, we connected the system to the solar energy system. We also improved the performance by fine tuning the PID controller and some features of the sensors and the control systems. Testing under various weather conditions showed that the system was capable of performing as intended. When the power input falls below activation levels, the system shuts the computer down until sufficient power is again available. The computer is reactivated when the power input exceeds a threshold.

The only major deviations in following the input power were cases in which the input power was beyond the limits of the computer (16 to 50 watts). Shutting down the computer was very fast when the power dropped. Reactivating the computing took a bit longer. During the periods when the computer is 
starting or shutting down its power usage cannot be controlled. To avoid frequent shutdowns and reactivations when there are rapid fluctuations in the input power, we used the average of the last minute values for decision making. This made the reaction power availability a bit slower but, on the other hand, avoided unnecessary changes in computing activity. Reducing idle power consumption has been widely studied in data centers. The benefit of shutting down servers depends on the applied method and used hardware [44,11,22]. In addition to totally shutting the server off, there are also several sleep states to be applied [36]. Although continuously switching on and off could reduce the lifetime of computer hardware, such an effect has not been noticed in these studies.

Another finding with longer test runs was that in weak sunlight conditions the accuracy of the sensors is important. To overcome this problem, the system was modified to use an external ADC converter in form of ATMega 644 chip. Furthermore, the measurement logic was changed to reduce errors in current measurements.

We also tested the system with emulated power production by disconnecting the solar panel and replaced it with a laboratory power supply which allowed controlling the available energy. For more extensive testing, we developed a generator to randomly vary the available power. The random generation algorithm varied voltage and current signals slightly all the time and over longer time intervals generated major fluctuations. This allowed us testing the system also in more extreme conditions than what was naturally available and repeating the tests with exactly the same power production profile after system modifications.

\subsection{System operation}

At the end of the integration testing, the system was capable of accurately matching the electricity consumption with the electricity production of the solar panels, as shown in Fig. 9.

In Fig. 9, we can see that when the power drops from $30 \mathrm{~W}$ to $20 \mathrm{~W}$ the system limits the $\mathrm{CPU}$ utilization of processes to achieve a matching reduction in power consumption. In this limited state, the system is still processing the workload but at reduced performance and throughput. When more power is again available, the control system lifts these limitations until power consumption matches the new power level.

When available power falls below the point in which no useful computations can be performed, the control system automatically shuts the computer down. In this state, the power consumption is nearly zero, allowing the system to preserve energy until suitable conditions for operating are met and computer can be restarted. There is a small but noticeable delay before the computer is shut down or restarted. However, with modern computing technology, these delays are and will become shorter and more manageable. 


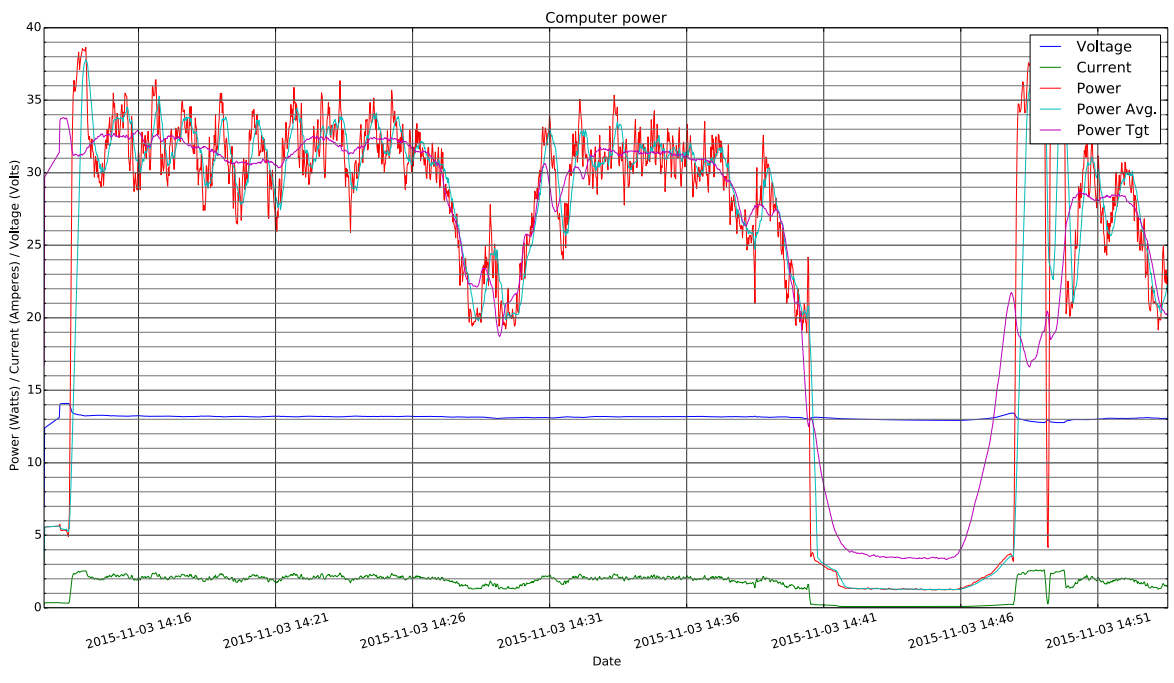

Fig. 9 Sample of systems operation in real conditions. The power values are on the top, the line in the middle represents voltage, and the lowest line is the current.

Although the power consumption is fluctuating around the target during the test, it is still close enough the target value. On the other hand, the computer should not drain the battery of the system excessively in the long run. In fact, improving the performance may be difficult as the power consumption may be influenced by components that are beyond the control of the system, such as memory and disk accesses. Any power drain caused by the fluctuations can be easily compensated by adjusting the consumption slightly below the given target.

The main issues with the system are mostly related to the nature of the PV panels and, in a small part, to the nature of the computer technology attached to the system. For the computer part, as mentioned earlier, there are inherent fluctuations in the power consumption due to the number of electrical components in the computer system and due to the limiting programs and the OS scheduling. Apart from these, the overhead in the computers is a minor problem that prevents the consumption of electricity only at very low levels. In case of the prototype system, the minimum power level at which activation of the system is viable was around 16 watts. At this level, the computer could be turned on, but very little useful calculations could be performed, since most of the power consumed at this level is spent on powering electronics on the motherboard and other system components. The result of this is that the system cannot efficiently utilize the excess energy when the power production is low.

This effect is partially enhanced by the nature of PV panels, which suffer power drop when even a small portion of the panel area is shadowed. Thus in low irradiance and situations where clouds or other objects cast even smallest of shadows to the panel area, the power production drops drastically and 
Table 6 Bill of material of the prototype

\begin{tabular}{|c|c|c|c|c|}
\hline \multicolumn{5}{|l|}{ PROTOTYPE COSTS } \\
\hline & Description & Amount & Single price & Total \\
\hline \multicolumn{5}{|l|}{ Control system } \\
\hline Intel Galileo Gen 1 & Development board & 1 & & $65 €$ \\
\hline LEM HXS 10-NP/SP3 & Current transducer & 2 & $17.56 €$ & $35.12 €$ \\
\hline Capacitors, Resistors, Wiring, etc. & Electronical components & - & & $10 €$ \\
\hline \multirow{2}{*}{ ATMega 644PU } & Microcontroller & 1 & $8.00 €$ & $8 €$ \\
\hline & & & Subtotal & $118 €$ \\
\hline \multicolumn{5}{|l|}{ Computer } \\
\hline Asus H97M-Plus & Motherboard & 1 & $109.90 €$ & $109.90 €$ \\
\hline Intel Core $13-4330$ & CPU & 1 & $154.90 €$ & $154.90 €$ \\
\hline Kingston DDR3 ValueRAM 2GB & Memory & 2 & $15.90 €$ & $31.80 €$ \\
\hline Kingston SSD V300 & Solid State Drive & 1 & $51.90 €$ & $51.90 €$ \\
\hline \multirow[t]{2}{*}{ CarTFT M4-ATX 250W PSU } & Power Supply & 1 & $84.95 €$ & $84.95 €$ \\
\hline & & & Subtotal & $433.45 €$ \\
\hline \multicolumn{5}{|l|}{ Solar system } \\
\hline 85 Watt SolarXon PV panel & Solar Panel & 1 & $139.00 €$ & $139.00 €$ \\
\hline EP-Solar Tracer 10A MPPT & Charge Controller & 1 & $119 €$ & $119 €$ \\
\hline Battery & Battery (46Ah) & 1 & $55 €$ & $55 €$ \\
\hline \multirow[t]{3}{*}{ Cables } & Power Cables & 20 meters & $20 €$ & $20 €$ \\
\hline & & & Subtotal & $333.00 €$ \\
\hline & & & Total & $885 €$ \\
\hline
\end{tabular}

suddenly. While the system can adapt to these changes quickly, the complete utilization of available electricity in these situations is difficult as usually the solar panels are unable to provide sufficient power to maintain the computer system. The upside of this is that the charge controller may still be able to generate sufficient voltage to provide charge for the battery, which compensates possible energy deficits caused by the system operation.

\subsection{Cost of the system}

As discussed above, the prototype shows that the system works. It can accurately track the energy influx from the solar panels and control the computer power consumption to match the amount of excess electricity. Next, in this subsection, we analyze the cost of building such a system.

Note that the costs are retail prices of the components in the shops we used to purchase the equipment. In industrial scale manufacturing, the prices would be considerable cheaper. Therefore, the main purpose of this subsection is to understand the relative costs of the different subsystems and identify the key components from the cost point of view. The bill of material of the prototype is presented in Table 6 . The costs are divided into three categories consisting of control, computer, and solar subsystems. 
The most expensive part of the prototype is by far the computer hardware covering over half of the project costs. In the calculation, we have also included the solar system but we assume that in a typical installation the solar system would already be present and we would only need to connect it with the computing and control subsystems. In that case, the computer system would dominate the costs even more clearly.

The computing subsystem costs are largely tied to the cost of computer hardware. In the prototype, we selected components that provided a great amount of reliability and flexibility, which was necessary for the easy development and testing. In future versions, the cost of components can be brought down by selecting less flexible components that meet the specific requirements of the system. In addition, in cases where several computing units are used, a more sensible approach is to equip only one computer with a disk drive and provide the operating system and storage to other computers using network booting.

For the part of the control system, the current system has a large initial cost, but scaling of the system is cheap because the ATMega and Galileo are able to measure additional sensors without modifications. Thus expansion of the system requires only addition of new sensors. Some further cost reductions can also be achieved by selecting a different embedded board to operate the system. It is also possible to find cheaper alternatives to Intel Galileo, which provide the needed features (Linux, Ethernet capability and GPIO).

\section{Discussion}

The prototype shows that the developed system can perform as expected and is relatively easy to build but the important question is the best use of this technology. The main assumption at the beginning was that the results of computing could be far more valuable than the electricity. Generally, research institutes are interested using a distributed computing platforms such as BOINC $[26,24,4]$. Other studies show that these platforms can be run over ad hoc networks [16]. In addition, so called many-task computing problems could also be suitable for our platform [43]. Therefore, testing the prototype using BOINC distributed projects as workload shows that calculations run in our solar-based system can be beneficial. However, determining the true monetary value of the data processing results is difficult. Possibly some kind of new business models would be needed for such intermittently available computing.

In Section 3 we estimated the value of the computation by comparing how much it would cost to rent or operate similar hardware in a cloud service such as Amazon EC2. Scaling those calculations to our prototype shows that its monthly computing could be worth tens of euros. The difficulty lies in the fact that most of the alternative computing services provide some kind of guarantees of their service levels. In our case there will be more fluctuations and randomness in the amount of available computing capacity. As a result only some computational tasks are feasible for this kind of setting. Moreover 
the computing hardware would only be active when enough solar power is available making the utilization level of the hardware investment quite low.

We are faced with several options on how to solve this dilemma. The first one would be to find services that are not time critical and the tasks need to be computed within a loose timeframe. That is, services similar to BOINC and the scientific computing. However, this approach does not solve the issue of low utilization: unless there is high availability of solar energy throughout the year, the computer system will be underutilized and some of its value generating potential will be lost.

The second approach is to partially combine the system to electric grid and use the grid to power the computers during the low solar irradiance months of the year. Still the main energy source would be the solar power. In addition, in such a setup, the computers could be used for the dual purpose of solar computing in the summertime and heating during the wintertime. Thus we could gain energy benefits both in the summer and in the winter by repurposing the computers. Computing would only be performed when heating is needed or excess electricity would be available. The problem with this approach is that we are still losing some of the potential of the computers and we still cannot provide services to true real-time applications throughout the year. The use for heating also means that the computers would have to be situated suitably to provide heat, which would mean that they would produce heat also during the summertime, which may pose a problem in some cases.

The third option is to use the computers constantly and use electricity from the grid as a complementary source when the solar electricity production is not adequate. With this approach, we can cater to most of the different service needs, but in part we drift farther from the original idea of using only excess electricity for computations. The end result of this would be a partially solar powered distributed computing system, that would consist of computers or servers located in households. These computers would perform centrally allocated tasks and use normal electricity grid as their primary energy source and solar energy whenever it is available. The benefit from using the electricity for computations in this manner is obvious, when looking at the monthly prices of computing and prices of electricity when bought or sold to the network. These show that, in theory, it would be possible to operate the computer profitably with only bought electricity. With the electricity supplied partly by the solar system, the electricity costs are further reduced. Finally, it is also possible to use other renewable energy sources, such as the wind power, in addition to the solar power. This would naturally increase the cost of the system but it would also improve the utilization level. Using a microgrid to connect several renewable energy resources would work well in this scenario.

\section{Conclusions}

In this work, we have discussed the idea of using excess electricity generated with solar or other means at homes for distributed computing. In this way, we 
would avoid many of the problems related to feeding the electricity back to grid. With today's tariff structures and computation prices (which vary a lot in different regions) the systems seems economically viable and even attractive.

We have developed a prototype system to test the idea in practice. The prototype shows that the concept is feasible and it is relatively easy to build out of regular electronic components. Our experience in building and testing the prototype has shown what kind of considerations are important for the smooth operation of the system. The prototype has also allowed us to estimate the building cost of such systems.

Assuming both computing needs and solar energy production in the world are continuously increasing, our Sunburn system could be one way to fulfill a part of the need of the computing and, at the same time, help in spending the excess energy. Since the computing power will fluctuate a lot as a function of available excess electricity, the system can only be used in cases which are not sensitive to computing delays. Plenty of tasks, for example in scientific computing, belong in this category. On the other hand, the operating time of the system can be increased by using electricity from the grid when excess energy is not available.

Like any new idea there is considerable uncertainty of the feasibility of the concept and its market acceptance. The technical risks seem minor but the risks of the business feasibility are high. Our calculations show that the concept can make economic sense but the calculations are based on a set of assumptions which need to be further tested and strengthened. In particular, the willingness of users to pay for this kind of service needs further investigation. The intermittently available computing power is one aspect that needs to be compensated in pricing. Moreover, the extreme distribution of computing may also be unacceptable for use cases where privacy or reliability requirements are high. Therefore, further analysis of the business side of the concept is an important area for further work. Our detailed analysis using a single country as a concrete example would also need to be extended to different geographical zones. Readers interested in exploring how the concept would work in different regions, and with different assumptions, can use our model, which, as described in Section 4, is accessible in the net.

The idea still needs a lot of refinement to reach a state for serious use. In particular, we would need a large scale prototype implementation with multiple computing units to understand how the system scales to the power levels of typical solar power installations. We would also need to explore better what kind of workloads would make sense in this environment, what would be the monetary value of those, and how it would be related to the investments needed to purchase the system.

\section{References}

1. Anderson, D.P.: Boinc: A system for public-resource computing and storage. In: Proceedings of the 5th IEEE/ACM International Workshop on Grid Computing, 
GRID '04, pp. 4-10. IEEE Computer Society, Washington, DC, USA (2004). DOI 10.1109/GRID.2004.14. URL http://dx.doi.org/10.1109/GRID.2004.14

2. Anderson, D.P., Cobb, J., Korpela, E., Lebofsky, M., Werthimer, D.: Seti@ home: an experiment in public-resource computing. Communications of the ACM 45(11), 56-61 (2002)

3. Anghel, I., Bertoncini, M., Cioara, T., Cupelli, M., Georgiadou, V., Jahangiri, P., Monti, A., Murphy, S., Schoofs, A., Velivassaki, T.: Geyser: Enabling green data centres in smart cities. In: International Workshop on Energy Efficient Data Centers, pp. 71-86. Springer (2014)

4. Asnicar, F., Sella, N., Masera, L., Morettin, P., Tolio, T., Semeniuta, S., Moser, C., Blanzieri, E., Cavecchia, V.: Tn-grid and gene@ home project: Volunteer computing for bioinformatics. In: Second international conference BOINC-based high performance computing: Fundamental research and development (BOINC: FAST 2015), 1502, pp. $1-15(2015)$

5. Binti, N.N., Zakaria, M.N.B., Aziz, I.B.A., Binti, N.S.: Optimizing boinc scheduling using genetic algorithm based on thermal profile. In: Computer and Information Sciences (ICCOINS), 2014 International Conference on, pp. 1-6. IEEE (2014)

6. Bird, S., Achuthan, A., Maatallah, O.A., Hu, W., Janoyan, K., Kwasinski, A., Matthews, J., Mayhew, D., Owen, J., Marzocca, P.: Distributed (green) data centers: A new concept for energy, computing, and telecommunications. Energy for Sustainable Development 19, 83 - 91 (2014). DOI http://dx.doi.org/10.1016/j.esd.2013.12.006. URL http:// www.sciencedirect.com/science/article/pii/S0973082613001129

7. Campoccia, A., Dusonchet, L., Telaretti, E., Zizzo, G.: An analysis of feed'in tariffs for solar pv in six representative countries of the european union. Solar Energy 107, $530-542(2014)$

8. de Carvalho Junior, O.A., Bruschi, S.M., Santana, R.H.C., Santana, M.J.: Green cloud meta-scheduling. Journal of Grid Computing 14(1), 109-126 (2016)

9. Cioara, T., Anghel, I., Bertoncini, M., Salomie, I., Arnone, D., Mammina, M., Velivassaki, T.H., Antal, M.: Optimized flexibility management enacting data centres participation in smart demand response programs. Future Generation Computer Systems pp. - (2016). DOI http://doi.org/10.1016/j.future.2016.05.010. URL http: //www.sciencedirect.com/science/article/pii/S0167739X16301200

10. CPUBoss: Compare CPUs to see which one is faster (2016). Http://cpuboss.com/cpus/Intel-Xeon-E5-2690-vs-Intel-Core-i3-4330 [Online; accessed 19-April-2016]

11. Deng, W., Liu, F., Jin, H., Liao, X., Liu, H., Chen, L.: Lifetime or energy: Consolidating servers with reliability control in virtualized cloud datacenters. In: Cloud Computing Technology and Science (CloudCom), 2012 IEEE 4th International Conference on, pp. 18-25. IEEE (2012)

12. Denholm, P., Margolis, R.M.: Evaluating the limits of solar photovoltaics (pv) in electric power systems utilizing energy storage and other enabling technologies. Energy Policy 35(9), 4424-4433 (2007)

13. Dunn, B., Kamath, H., Tarascon, J.M.: Electrical energy storage for the grid: a battery of choices. Science 334(6058), 928-935 (2011)

14. Dupont, C., Hermenier, F., Schulze, T., Basmadjian, R., Somov, A., Giuliani, G.: Plug4green: A flexible energy-aware vm manager to fit data centre particularities. Ad Hoc Networks 25, 505-519 (2015)

15. Dupont, C., Sheikhalishahi, M., Facca, F.M., Cretti, S.: Energy efficient data centres within smart cities: Iaas and paas optimizations. In: Smart City 360, pp. 408-415. Springer (2016)

16. Funai, C., Tapparello, C., Ba, H., Karaoglu, B., Heinzelman, W.: Extending volunteer computing through mobile ad hoc networking. In: Global Communications Conference (GLOBECOM), 2014 IEEE, pp. 32-38. IEEE (2014)

17. Garcia-Valls, M., Bellavista, P., Gokhale, A.: Reliable software technologies and communication middleware: A perspective and evolution directions for cyber-physical system, mobility, and cloud computing. Future Generation Computer Systems 71, 171 - 176 (2017). DOI http://doi.org/10.1016/j.future.2017.02.037. URL http://www . sciencedirect.com/science/article/pii/S0167739X17302819 
18. García-Valls, M., Calva-Urrego, C.: Improving service time with a multicore aware middleware Proceedings of the Symposium on Applied Computing (SAC). Marrakech, Morocco. April 2017, pp. 1548-1553. ACM (2017)

19. Garcia-Valls, M., Cucinotta, T., Lu, C.: Challenges in real-time virtualization and predictable cloud computing. Journal of Systems Architecture 60(9), $726-740$ (2014). DOI http://doi.org/10.1016/j.sysarc.2014.07.004. URL http://www.sciencedirect. com/science/article/pii/S1383762114001015

20. Goiri, Í., Katsak, W., Le, K., Nguyen, T.D., Bianchini, R.: Parasol and greenswitch: managing datacenters powered by renewable energy. In: ACM SIGARCH Computer Architecture News, vol. 41, pp. 51-64. ACM (2013)

21. Gottwalt, S., Ketter, W., Block, C., Collins, J., Weinhardt, C.: Demand side management - a simulation of household behavior under variable prices. Energy Policy 39(12), 8163-8174 (2011)

22. Guenter, B., Jain, N., Williams, C.: Managing cost, performance, and reliability tradeoffs for energy-aware server provisioning. In: 2011 Proceedings IEEE INFOCOM, pp. 13321340 (2011). DOI 10.1109/INFCOM.2011.5934917

23. Heddeghem, W.V., Vereecken, W., Colle, D., Pickavet, M., Demeester, P.: Distributed computing for carbon footprint reduction by exploiting low-footprint energy availability. Future Generation Computer Systems 28(2), 405 - 414 (2012). DOI http://dx.doi.org/ 10.1016/j.future.2011.05.004. URL http://www.sciencedirect.com/science/article/ pii/S0167739X11000859

24. Høimyr, N., Marquina, M., Asp, T., Jones, P., Gonzalez, A., Field, L.: Towards a production volunteer computing infrastructure for hep. In: Journal of Physics: Conference Series, vol. 664, p. 022023. IOP Publishing (2015)

25. Hoppmann, J., Huenteler, J., Girod, B.: Compulsive policy-making - the evolution of the german feed-in tariff system for solar photovoltaic power. Research policy 43(8), 1422-1441 (2014)

26. Jenviriyakul, P., Chalumporn, G., Achalakul, T., Costa, F., Akkarajitsakul, K.: Alice connex: A volunteer computing platform for alice experiments. In: Big Data and Smart Computing (BigComp), 2016 International Conference on, pp. 300-303. IEEE (2016)

27. Kommeri, J., Niemi, T., Nurminen, J.K.: Energy efficiency of dynamic management of virtual cluster with heterogeneous hardware. The Journal of Supercomputing pp. 1-23 (2016)

28. Kong, F., Liu, X.: A survey on green-energy-aware power management for datacenters. ACM Computing Surveys 47(2) (2014). DOI 10.1145/2642708. URL http://www.scopus.com/inward/record.url?eid=2-s2.0-84911886554\&partnerID= $40 \& m d 5=d 585871 \mathrm{f} 5107592 \mathrm{f} \mathrm{c} 6 \mathrm{~b} 6 \mathrm{~b} 7663 \mathrm{~b} 47 \mathrm{a} 1 \mathrm{ab}$. Cited By 1

29. Koomey, J.: Growth in data center electricity use 2005 to 2010. A report by Analytical Press, completed at the request of The New York Times p. 9 (2011)

30. Kriett, P.O., Salani, M.: Optimal control of a residential microgrid. Energy 42(1), 321-330 (2012)

31. Larcher, D., Tarascon, J.: Towards greener and more sustainable batteries for electrical energy storage. Nature chemistry 7(1), 19-29 (2015)

32. Le, T., Wright, D.: Scheduling workloads in a network of datacentres to reduce electricity cost and carbon footprint. Sustainable Computing: Informatics and Systems 5, 31 40 (2015). DOI http://dx.doi.org/10.1016/j.suscom.2014.08.012. URL http://www . sciencedirect.com/science/article/pii/S2210537914000535

33. Lidula, N., Rajapakse, A.: Microgrids research: A review of experimental microgrids and test systems. Renewable and Sustainable Energy Reviews 15(1), 186-202 (2011)

34. Liu, J., Goraczko, M., James, S., Belady, C., Lu, J., Whitehouse, K.: The data furnace: Heating up with cloud computing. In: 3rd USENIX Workshop on Hot Topics in Cloud Computing. USENIX (2011). research.microsoft.com/apps/pubs/default.aspx?id= 150265

35. Lund, H., Marszal, A., Heiselberg, P.: Zero energy buildings and mismatch compensation factors. Energy and Buildings 43(7), 1646-1654 (2011)

36. Meisner, D., Gold, B.T., Wenisch, T.F.: Powernap: eliminating server idle power. In: ACM Sigplan Notices, vol. 44, pp. 205-216. ACM (2009) 
37. Mukherjee, T., Dutta, P., Hegde, V.G., Gujar, S.: Risc: Robust infrastructure over shared computing resources through dynamic pricing and incentivization. In: Parallel and Distributed Processing Symposium (IPDPS), 2015 IEEE International, pp. 11071116. IEEE (2015)

38. Mundada, A.S., Shah, K.K., Pearce, J.: Levelized cost of electricity for solar photovoltaic, battery and cogen hybrid systems. Renewable and Sustainable Energy Reviews 57, 692 - 703 (2016). DOI http://dx.doi.org/10.1016/j.rser.2015.12.084. URL http://www.sciencedirect.com/science/article/pii/S1364032115014677

39. Nurminen, J.K., Strandman, J., Koskela, K., Niemi, T.: Computing Heaters - An Energy-efficient Way to Provide Computing Services. ExtremeGreen 2015: Extreme Green \& Energy Efficiency in Large Scale Distributed Systems, May 4-7, Shenzen, Guangdong, China (2015).

40. Ou, Z., Zhuang, H., Lukyanenko, A., Nurminen, J.K., Hui, P., Mazalov, V., Yla-Jaaski, A.: Is the same instance type created equal? exploiting heterogeneity of public clouds. Cloud Computing, IEEE Transactions on 1(2), 201-214 (2013)

41. Pernici, B., Cappiello, C., Fugini, M.G., Plebani, P., Vitali, M., Salomie, I., Cioara, T. Anghel, I., Henis, E., Kat, R., et al.: Setting energy efficiency goals in data centers: the games approach. In: International Workshop on Energy Efficient Data Centers, pp. 1-12. Springer (2012)

42. Rahman, A., Liu, X., Kong, F.: A survey on geographic load balancing based data center power management in the smart grid environment. IEEE Communications Surveys and Tutorials 16(1), 214-233 (2014). DOI 10.1109/SURV.2013.070813. 00183. URL http://www.scopus.com/inward/record.url?eid=2-s2.0-84894645914\& partnerID $=40 \& m d 5=f 447 b 4 a 15549 b f 59 c c 9 c f f 07 b 209 f c b 5$. Cited By 13

43. Raicu, I., Foster, I.T., Zhao, Y.: Many-task computing for grids and supercomputers. In: Many-Task Computing on Grids and Supercomputers, 2008. MTAGS 2008. Workshop on, pp. 1-11. IEEE (2008)

44. Raïs, I., Orgerie, A.C., Quinson, M.: Impact of shutdown techniques for energy-efficient cloud data centers. In: Algorithms and Architectures for Parallel Processing, pp. 203210. Springer (2016)

45. Rodrigues, S., Faria, F., Ivaki, A.R., Cafofo, N., Chen, X., Morgado-Dias, F.: Tesla powerwall: Analysis of its use in portugal and united states. International Journal of Power and Energy Systems 36(1) (2016)

46. Shehabi, A., Smith, S.J., Sartor, D.A., Brown, R.E., Herrlin, M., Koomey, J.G., Masanet, E.R., Horner, N., Azevedo, I.L., Lintner, W.: United states data center energy usage report. Lawrence Berkeley National Laboratory, Berkeley, California. LBNL$1005775(2016)$

47. Shen, Z., Jia, Q., Sela, G.E., Rainero, B., Song, W., van Renesse, R., Weatherspoon, H.: Follow the sun through the clouds: Application migration for geographically shifting workloads. In: Proceedings of the Seventh ACM Symposium on Cloud Computing, SoCC '16, pp. 141-154. ACM, New York, NY, USA (2016). DOI 10.1145/2987550.2987561. URL http://doi.acm.org/10.1145/2987550.2987561

48. Stetz, T., Marten, F., Braun, M.: Improved low voltage grid-integration of photovoltaic systems in germany. IEEE Transactions on sustainable energy 4(2), 534-542 (2013)

49. Subramanya, S., Guo, T., Sharma, P., Irwin, D., Shenoy, P.: Spoton: A batch computing service for the spot market. In: Proceedings of the Sixth ACM Symposium on Cloud Computing, pp. 329-341. ACM (2015)

50. Trefke, J., Rohjans, S., Uslar, M., Lehnhoff, S., Nordstrom, L., Saleem, A.: Smart grid architecture model use case management in a large european smart grid project. In: Innovative Smart Grid Technologies Europe (ISGT EUROPE), 2013 4th IEEE/PES, pp. 1-5. IEEE (2013)

51. Truong, C.N., Naumann, M., Karl, R.C., Müller, M., Jossen, A., Hesse, H.C.: Economics of residential photovoltaic battery systems in germany: The case of tesla's powerwall. Batteries 2(2), 14 (2016)

52. Ujhelyi, M., Lacko, P., Paulovič, A.: Task scheduling in distributed volunteer computing systems. In: Intelligent Systems and Informatics (SISY), 2014 IEEE 12th International Symposium on, pp. 111-114. IEEE (2014) 
53. Valls, M.G., Lopez, I.R., Villar, L.F.: iLAND: An enhanced middleware for real-time reconfiguration of service oriented distributed real-time systems. IEEE Transactions on Industrial Informatics 9(1), 228-236 (2013). DOI 10.1109/TII.2012.2198662

54. Valls, M.G., Val, P.B.: Usage of dds data-centric middleware for remote monitoring and control laboratories. IEEE Transactions on Industrial Informatics 9(1), 567-574 (2013). DOI 10.1109/TII.2012.2211028

55. Walling, R.A., Saint, R., Dugan, R.C., Burke, J., Kojovic, L.A.: Summary of distributed resources impact on power delivery systems. IEEE Transactions on Power Delivery 23(3), 1636-1644 (2008). DOI 10.1109/TPWRD.2007.909115

56. Yang, F., Chien, A.A.: Zccloud: Exploring wasted green power for high-performance computing. IPDPS 2016 (2016)

57. Ye, R., Li, C., Liang, X.: Energy storage system in green data centers: Architecture and management. Jisuanji Yanjiu yu Fazhan/Computer Research and Development 53(2), 326-340 (2016). DOI 10.7544/issn1000-1239.2016.20150296. URL http://www.scopus.com/inward/record.url?eid=2-s2.0-84959886487\&partnerID= 40\&md5 $=57$ e1cc6e448105f766c77ee72bd9d1ef. Cited By 0

58. Zakeri, B., Syri, S.: Electrical energy storage systems: A comparative life cycle cost analysis. Renewable and Sustainable Energy Reviews 42, 569 - 596 (2015). DOI http:// dx.doi.org/10.1016/j.rser.2014.10.011. URL http://www.sciencedirect.com/science/ article/pii/S1364032114008284

59. Zheng, X., Cai, Y.: Energy-aware load dispatching in geographically located internet data centers. Sustainable Computing: Informatics and Systems 1(4), 275 285 (2011). DOI http://dx.doi.org/10.1016/j.suscom.2011.06.002. URL http://www . sciencedirect.com/science/article/pii/S221053791100045X 